\title{
Crossing-Critical Graphs and Path-Width
}

\author{
Petr Hliněný* \\ School of Mathematical and Computing Sciences, \\ Victoria University, \\ P.O. Box 600, Wellington, New Zealand, \\ and \\ Institute for Theoretical Computer Science ${ }^{\star \star}$ (ITI MFF), \\ Charles University, \\ Malostranské nám. 25, 11800 Praha 1, Czech Republic. \\ (e-mail: hlineny@member.ams.org , fax: +64-4-4635045)
}

\begin{abstract}
The crossing number $\operatorname{cr}(\boldsymbol{G})$ of a graph $\boldsymbol{G}$, is the smallest possible number of edge-crossings in a drawing of $\boldsymbol{G}$ in the plane. A graph $\boldsymbol{G}$ is crossing-critical if $\operatorname{cr}(\boldsymbol{G}-e)<\operatorname{cr}(\boldsymbol{G})$ for all edges $e$ of $\boldsymbol{G}$. G. Salazar conjectured in 1999 that crossing-critical graphs have pathwidth bounded by a function of their crossing number, which roughly means that such graphs are made up of small pieces joined in a linear way on small cut-sets. That conjecture was recently proved by the author [9]. Our paper presents that result together with a brief sketch of proof ideas. The main focus of the paper is on presenting a new construction of crossing-critical graphs, which, in particular, gives a nontrivial lower bound on the path-width. Our construction may be interesting also to other areas concerned with the crossing number.
\end{abstract}

\section{Introduction}

In this section we informally introduce the problem and our contributions to it. The reader is referred to the next section for formal definition and statements.

We are interested in drawing of (nonplanar) graphs in the plane that have a small number of edge-crossings. There are many practical applications of such drawings, including VLSI design 3, or graph visualization 4.14. Crossingnumber problems are often discussed on Graph Drawing conferences, recently for example [12,18,14].

Determining the crossing number of a graph is a hard problem [6] in general, and the crossing number is not even known exactly for complete or complete bipartite graphs. A lot of work has been done investigating the crossing number of particular graph classes like $C_{m} \times C_{n}$, see 15168 . For general graphs, research so far focused mainly on relations of the crossing number to nonstructural graph

* The research was partially supported by a New Zealand Marsden Fund research grant to Geoff Whittle, and by a Czech research grant GAČR 201/99/0242.

** Supported by the Ministry of Education of Czech Republic as project LN00A056.

P. Mutzel, M. Jünger, and S. Leipert (Eds.): GD 2001, LNCS 2265, pp. 102-114, 2002.

(C) Springer-Verlag Berlin Heidelberg 2002 
properties like the number of edges, for example 11113. On the other hand, crossing-critical graphs play a key role in investigation of structural properties of the crossing number. Our result tries to give some insight to the general structure of crossing-critical graphs, about which is not much known yet.

In Section 2 we state that if $\boldsymbol{G}$ is a $k$-crossing-critical graph, then $\boldsymbol{G}$ cannot contain a subdivision of a "large in $k$ " binary tree. It is known [17] that the latter condition is equivalent to $\boldsymbol{G}$ having "bounded in $k$ path-width", which roughly means that $\boldsymbol{G}$ is made up of small pieces joined in a linear way on small cut-sets. We also sketch basic proof ideas for this result in Section 3, while the whole proof (which is rather long) can be found in 9 .

We mainly focus on constructions of crossing-critical graphs that give good lower bounds on the path-width (in terms of binary trees) in Section 4 Specifically, we present new general classes of $k$-crossing-critical graphs for $k \geq 3$, and we prove their values of the crossing number. These classes contain graphs with binary trees of heights up to $k+2$. We think that these classes may be also interesting to other areas concerned with the crossing number.

\section{$2 \quad$ Definitions and Results}

We consider finite simple graphs in the paper. We usually speak about actual drawings of graphs instead of abstract graphs here. If $\varrho:[0,1] \rightarrow \mathbb{R}^{2}$ is a simple continuous function, then $\varrho([0,1])$ is a simple curve, and $\varrho((0,1))$ is a simple open curve.

Definition. A graph $\boldsymbol{G}$ is drawn in the plane if the vertices of $\boldsymbol{G}$ are distinct points of $\mathbb{R}^{2}$, and every edge $e=u v \in E(\boldsymbol{G})$ is a simple open curve $\varrho$ such that $\varrho(0)=u, \varrho(1)=v$. Moreover, it is required that no edge contains a vertex of $\boldsymbol{G}$, and that no three distinct edges of $\boldsymbol{G}$ share a common point. An (edge-) crossing is any point of the drawing that belongs to two distinct edges.

(Notice that our edge as a topological object does not include its endpoints. In particular, when we speak about a crossing, we do not mean a common end of two edges.)

Definition. The crossing number $\operatorname{cr}(\boldsymbol{G})$ of a graph $\boldsymbol{G}$ is the smallest possible number of edge-crossings in a drawing of $\boldsymbol{G}$ in the plane. A graph $\boldsymbol{G}$ is crossingcritical if $\operatorname{cr}(\boldsymbol{G}-e)<\operatorname{cr}(\boldsymbol{G})$ for all edges $e \in E(\boldsymbol{G})$. A graph $\boldsymbol{G}$ is $k$-crossingcritical if $\boldsymbol{G}$ is crossing-critical and $\operatorname{cr}(\boldsymbol{G})=k$.

The crossing number stays the same if we consider drawings on the sphere instead of the plane, or if we require piecewise-linear drawings. (However, if we require the edges to be straight segments - so called rectilinear crossing number, we get completely different behavior; but we are not dealing with this concept here.) Also, the crossing number is clearly preserved under subdivisions of edges (although not under contractions). Thus it is not an essential restriction when we consider simple graphs only. 
One annoying thing about the crossing number is that there exist other possible definitions of it, and we do not know whether they are all equivalent or not. The pairwise-crossing number $\mathrm{cr}_{\text {pair }}$ is defined similarly, but it counts the number of crossing pairs of edges, instead of crossing points. The odd-crossing number $\mathrm{cr}_{\mathrm{odd}}$ counts the number of pairs of edges that cross odd number of times only. It clearly follows that $\operatorname{cr}_{\text {odd }}(\boldsymbol{G}) \leq \mathrm{cr}_{\text {pair }}(\boldsymbol{G}) \leq \operatorname{cr}(\boldsymbol{G})$, and it was proved by Tutte [19] that $\mathrm{cr}_{\text {odd }}(\boldsymbol{G})=0$ implies $\operatorname{cr}(\boldsymbol{G})=0$. The best known general relation between these crossing numbers is due to Pach and Tóth [13 who proved $\operatorname{cr}(\boldsymbol{G}) \leq 2 \mathrm{cr}_{\text {odd }}(\boldsymbol{G})^{2}$. Our results are formulated for the ordinary crossing number, however, they hold as well for the pairwise-crossing number.

Further we define the path-width of a graph and present its basic properties. A notation $\boldsymbol{G} \uparrow X$ is used for the subgraph of $\boldsymbol{G}$ induced by the vertex set $X$. A minor is a graph obtained from a subgraph by contractions of edges.

Definition. A path decomposition of a graph $\boldsymbol{G}$ is a sequence of sets $\left(W_{1}, W_{2}\right.$, $\left.\ldots, W_{p}\right)$ such that $\bigcup_{1 \leq i \leq p} W_{i}=V(\boldsymbol{G}), \quad \bigcup_{1 \leq i \leq p} E\left(\boldsymbol{G} \uparrow W_{i}\right)=E(\boldsymbol{G})$, and $W_{i} \cap W_{k} \subseteq W_{j}$ for all $1 \leq i<j<k \leq p$. The width of a path decomposition is $\max \left\{\left|W_{i}\right|-1: 1 \leq i \leq p\right\}$. The path-width of a graph $\boldsymbol{G}$, denoted by $\operatorname{pw}(\boldsymbol{G})$, is the smallest width of a path decomposition of $\boldsymbol{G}$.

It is known [17] that if $\boldsymbol{G}$ is a minor of $\boldsymbol{H}$, then $\operatorname{pw}(\boldsymbol{G}) \leq \operatorname{pw}(\boldsymbol{H})$. A binary tree of height $h$ a rooted tree $\boldsymbol{T}$ such that the root has degree 2, all other nonleaf vertices of $\boldsymbol{T}$ have degrees 3, and every leaf of $\boldsymbol{T}$ has distance $h$ from the root. (A binary tree of height $h$ has $2^{h+1}-1$ vertices.) Since the maximal degree of a binary tree $\boldsymbol{T}$ is 3, a graph $\boldsymbol{H}$ contains $\boldsymbol{T}$ as a minor if and only if $\boldsymbol{H}$ contains $\boldsymbol{T}$ as a subdivision. The important connection between binary trees and path-width was first established by Robertson and Seymour in [17], while the following strengthening is due to [2]:

Theorem 2.1. (Bienstock, Robertson, Seymour, Thomas)

(a) If $\boldsymbol{T}$ is a binary tree of height $h$, then $\operatorname{pw}(\boldsymbol{T}) \geq \frac{h}{2}$.

(b) If $\mathrm{pw}(\boldsymbol{G}) \geq p$, then $\boldsymbol{G}$ contains any tree on $p$ vertices as a minor.

We look closer at some facts about crossing-critical graphs. By the Kuratowski theorem, there are only two 1-crossing-critical graphs $\boldsymbol{K}_{5}$ and $\boldsymbol{K}_{3,3}$, up to subdivisions. On the other hand, an infinite family of 2-crossing-critical graphs with minimal degree more than 2 was found by Kochol in [10]. One may easily observe that every edge-transitive graph is crossing-critical, while the converse is not true, of course.

Ding, Oporowski, Thomas and Vertigan [5] have proved that every 2-crossingcritical graph satisfying certain simple assumptions and having sufficiently many vertices belongs to a well-defined infinite graph class. In particular, these graphs have bounded path-width. Analyzing the structure of other known infinite classes of crossing-critical graphs, G. Salazar formulated the following conjecture, appearing in [7]. 
Conjecture 2.2. (Salazar, 1999) There exists a function $g$ such that any $k$ crossing-critical graph has path-width at most $g(k)$.

The paper [7] proves a weaker statement that the tree-width of a crossingcritical graph is bounded. Our Theorem [2.3 9], together with Theorem 2.1. immediately imply a solution to Salazar's conjecture.

Theorem 2.3. There exists a function $f$ such that no $k$-crossing-critical graph contains a subdivision of a (complete) binary tree of height $f(k)$. In particular, $f(k) \leq 6 \cdot\left(72 \log _{2} k+248\right) \cdot k^{3}$.

Corollary 2.4. Let $f$ be the function from Theorem 2.3. If $\boldsymbol{G}$ is a $k$-crossingcritical graph, then the path-width of $\boldsymbol{G}$ is at most $2^{f(k)+1}-2$.

Remark. It is important that Theorem 2.3 speaks about crossing-critical graphs, since an arbitrary graph of a fixed crossing number $k$ may contain a binary tree of any height. There is no direct connection between the crossing number and the path-width of a graph without an assumption of being crossingcritical.

A natural question arises about lower bounds on the function $f$ from Theorem 2.3. An easy argument shows that $f(k)$ must grow with $k$ : The complete graph $\boldsymbol{K}_{n}$ is crossing-critical for $n \geq 5$ with the crossing number growing roughly as $\Theta\left(n^{4}\right)$, and $\boldsymbol{K}_{n}$ contains a binary tree of height $\left\lfloor\log _{2} n\right\rfloor-1$. (In fact, the pathwidth of $K_{n}$ is $n-1$.) However, we are able to provide much better bounds on $f$ as consequences of a general construction presented in Section 4

Theorem 2.5. Let $f$ be the function from Theorem 2.3, and $k \geq 3$. Then $f(k) \geq$ $k+3$, or $f(k) \geq k$ if we consider only simple 3 -connected graphs.

\section{Upper Bound Sketch}

The whole proof [9] of Theorem 2.3 is quite long, so here we present only an informal short sketch of it. Suppose that $\boldsymbol{G}$ is a graph drawn in the plane with $k$ crossings. The basic idea behind our proof is that if sufficiently many nested edge-disjoint cycles "separate" all crossed edges from some edge $e$ in $\boldsymbol{G}$, then $\boldsymbol{G}$ cannot be crossing-critical since deleting $e$ cannot decrease its crossing number. (This trick was suggested earlier by Salazar in connection with the tree-width of crossing-critical graphs.) Unfortunately, considering sequences of single cycles is not enough to achieve our goal. So we actually work with so called "nesting" and "cutting" sequences in the graph $\boldsymbol{G}$ (see Lemmas 3.1 and 3.2).

Recall that $\boldsymbol{G}$ is a graph drawn in the plane. Informally speaking, a multicycle $M$ in $\boldsymbol{G}$ is a collection of (not necessarily disjoint) cycles of $\boldsymbol{G}$ such that no two of these cycles are crossed or nested. (These words implicitly refer to the infinite face of the drawing.) The finite faces bounded by the cycles of $M$ are called the 
interior faces of $M$. We say that a multicycle $M$ is nested in a multicycle $M^{\prime}$, denoted by $M \preceq M^{\prime}$, if each interior face of $M$ is contained in some interior face of $M^{\prime}$. We say that $M$ is strictly nested in $M^{\prime}$, denoted by $M \prec M^{\prime}$, if $M \preceq M^{\prime}$, and if $M$ and $M^{\prime}$ share at most one vertex. See an illustration in Fig. 1

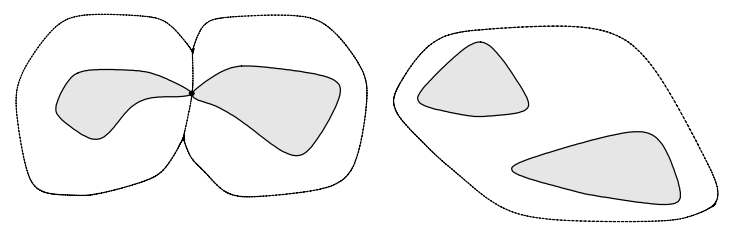

Fig. 1. An example of two strictly nested multicycles $M \nVdash M^{\prime}$ (shaded $M$ consists of 4 cycles, and $M^{\prime}$ consists of 3 cycles).

Let $M_{1} \preccurlyeq M_{2} \preccurlyeq \ldots \nVdash M_{c}$ be a sequence of $c$ strictly nested multicycles in the graph $\boldsymbol{G}$. Suppose that all crossed edges of $\boldsymbol{G}$ are contained in the interior faces of $M_{1}$, and that, for each interior face $\Phi$ of $M_{i}, 2 \leq i \leq c$, every component of the subgraph of $\boldsymbol{G}$ drawn inside $\Phi$ intersects some cycle of $M_{i-1}$ in $\Phi$. Then $\mathcal{M}_{c}(\boldsymbol{G})=\left(M_{1}, \ldots, M_{c}\right)$ is called a c-nesting sequence in $\boldsymbol{G}$.

Lemma 3.1. Suppose that there exists a $(3 k-1)$-nesting sequence in a 2 connected graph $\boldsymbol{H}$ drawn in the plane. Then $\boldsymbol{H}$ is not $k$-crossing-critical.

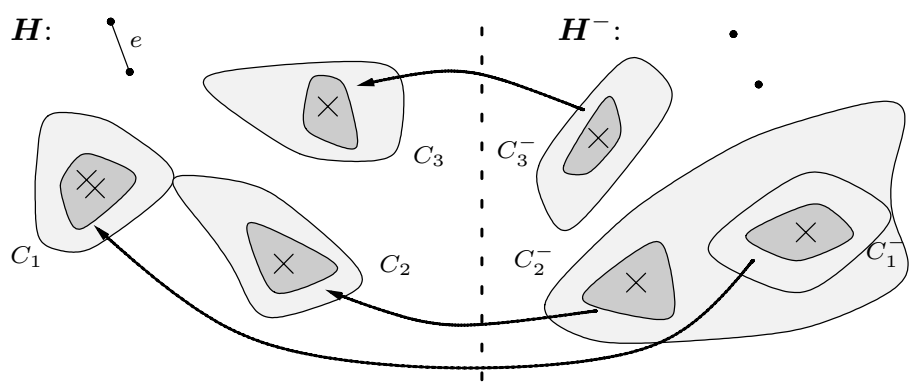

Fig. 2. An illustration to Lemma 3.1 how a better drawing of $\boldsymbol{H}$ is obtained using parts of the drawing $\boldsymbol{H}^{-} \simeq \boldsymbol{H}-e$ that has fewer than $k$ crossings.

Proof. Let $\mathcal{M}_{3 k-1}(\boldsymbol{H})=\left(M_{1}, \ldots, M_{3 k-1}\right)$ be a $(3 k-1)$-nesting sequence in $\boldsymbol{H}$. Briefly speaking, our goal is to delete an edge $e$ in the exterior of $M_{3 k-1}$, draw the new graph with fewer crossings, and use pieces of the new drawing to "improve" the drawing $\boldsymbol{H}$. Notice that $M_{1}$ consists of at most $k$ cycles (one for each crossings), and that the number of cycles does not increase in the sequence.

If $\boldsymbol{H}$ is $k$-crossing-critical, then there exists a drawing $\boldsymbol{H}^{-}$of the graph $\boldsymbol{H}-e$ with fewer than $k$ crossings. We denote by $M_{1}^{-}, \ldots, M_{3 k-1}^{-}$the corresponding 
multicycles in $\boldsymbol{H}^{-}$. One edge-crossing may involve at most two multicycles, so at least $k$ multicycles of $M_{2}^{-}, \ldots, M_{3 k-1}^{-}$are not crossed in $\boldsymbol{H}^{-}$. Thus there exists an index $2 \leq i \leq 3 k-1$ such that $M_{i}^{-}$is not crossed, and that $M_{i}^{-}, M_{i-1}^{-}$consist of the same number $m$ of cycles. Let $\boldsymbol{H}_{j}, j=1, \ldots, m$ be the subgraph of $\boldsymbol{H}$ drawn inside the $j$-th cycle of $M_{i}$, and let $\boldsymbol{H}_{j}^{-}$be the corresponding subgraph in $\boldsymbol{H}^{-}$. The graphs $\boldsymbol{H}_{j} \simeq \boldsymbol{H}_{j}^{-}$are connected by the definition. Since the multicycle $M_{i}^{-}$in $\boldsymbol{H}^{-}$is not crossed, we may "cut" the subdrawings $\boldsymbol{H}_{j}^{-}$and "paste" them into the interior faces of $M_{i}$ in $\boldsymbol{H}$ instead of $\boldsymbol{H}_{j}, j=1, \ldots, m$. Recall that all crossings of $\boldsymbol{H}$ belonged to some $\boldsymbol{H}_{j}$. Therefore, the new drawing of $\boldsymbol{H}$ has at most as many crossings as $\operatorname{cr}\left(\boldsymbol{H}^{-}\right)<k$, a contradiction.

We say that a sequence $P_{1}, \ldots, P_{q}$ of pairwise disjoint paths in a graph $\boldsymbol{G}$ is a $q$-cutting sequence if each set $V\left(P_{i}\right)$ is a cut in $\boldsymbol{G}$ separating $X \cup P_{1} \cup \ldots \cup P_{i-1}$ from $P_{i+1} \cup \ldots \cup P_{q}$, where $X$ is a subgraph formed by all crossed edges of $\boldsymbol{G}$. Similarly as in the previous lemma we prove:

Lemma 3.2. Suppose that there exists a $4 k$-cutting sequence in a 2-connected graph $\boldsymbol{H}$ drawn in the plane. Then $\boldsymbol{H}$ cannot be $k$-crossing-critical.

Finally, the lengthy part of the proof of Theorem 2.3 comes in. We want to show that a 2-connected graph with a sufficiently large binary tree contains a long nesting or cutting sequence. Obviously, if our graph $\boldsymbol{H}$ is not 2-connected, we may prove the theorem separately for the blocks of $\boldsymbol{H}$.

Lemma 3.3. Let $\boldsymbol{H}$ be a 2-connected graph that is drawn in the plane with $k$ crossings. Suppose that $\boldsymbol{H}$ contains a subdivision of a binary tree of height $6 \cdot\left(72 \log _{2} k+248\right) \cdot k^{3}$. Then there exists a $(3 k-1)$-nesting sequence or a $4 k$ cutting sequence in $\boldsymbol{H}$.

To prove the lemma, we try to inductively construct a $c$-nesting sequence in $\boldsymbol{H}$ for $c=1,2, \ldots, 3 k-1$, such that the multicycles in the sequence satisfy certain rather complicated connectivity property, and that a "large portion" of the subdivision of a binary tree in $\boldsymbol{H}$ stays outside of the sequence. Let us denote by $f^{\prime}(k)=\left(72 \log _{2} k+248\right) k^{2}$, by $f(k)=6 k f^{\prime}(k)$, and by $f_{i}(k)=$ $(6 k-2 i-1) f^{\prime}(k)$. The first multicycle $M_{1}$ of the sequence encloses all crossed edges of $\boldsymbol{H}$, and, at each step $c$, there is a subdivision $\boldsymbol{U} \subset \boldsymbol{H}$ of a binary tree of height $f_{c}(k)$ drawn in the infinite face of the last multicycle $M_{c}$. For simplicity, say that $\boldsymbol{U}$ actually is a binary tree.

Now we briefly describe a single step of our construction. We divide the binary tree $\boldsymbol{U}$ of height $f_{c}(k)$ into "layers" of heights $f^{\prime}(k), f^{\prime}(k)$, and $f_{c+1}(k)$. (For example, a subtree of $\boldsymbol{U}$ in the "middle layer" has its root at distance $f^{\prime}(k)$ and its leaves at distance $2 f^{\prime}(k)$ from the root of $\boldsymbol{U}$.)

- First we look whether the leaves of some middle-layer subtree of $\boldsymbol{U}$ are "surrounded" by a common face of $\boldsymbol{H}$. If this happens, then either there is a next multicycle $M_{c+1}$ for our nesting sequence (using part of boundary of the common face), or selected paths of the mentioned subtree form a $4 k$-cutting sequence. 
- If we are not successful in the previous step, then we argue that most of the middle-layer subtrees are "cut in half" by closed curves in the drawing $\boldsymbol{H}$. If sufficiently many of such curves do not intersect $M_{c}$, then they form many graph cycles in $\boldsymbol{H}$. We use the cycles to construct a multicycle $M_{c+1}$ such that some of the bottom-layer subtrees of $\boldsymbol{U}$ of height $f_{c+1}(k)$ stays in the infinite face of $M_{c+1}$.

- Otherwise, most of middle-layer subtrees are connected by pairwise disjoint paths to vertices of $M_{c}$. In such case we apply the above mentioned connectivity property of our sequence (which is specifically tailored to solve this case); and using the connecting paths, we construct another $(3 k-1)$-nesting or $4 k$-cutting sequence in $\boldsymbol{H}$ straight away.

We skip the details of this proof here.

\section{4 "Crossed-Fence" Construction}

Let $k$ be a positive integer. We describe a graph class parametrized by $k$, and we later prove that the graphs from this class are $k$-crossing-critical. (The name "fence" for the class was chosen by resemblance of the example from Fig. 3)

Definition. Let $C_{1}, C_{2}, \ldots, C_{k}$ be a sequence of some $k$ edge-disjoint graph cycles, let $\boldsymbol{F}_{0}=C_{1} \cup C_{2} \cup \ldots \cup C_{k}$ be a graph, and let $u_{1}, u_{2} \in V\left(C_{1}\right), u_{3}, u_{4} \in V\left(C_{k}\right)$. The 5-tuple $\left(\boldsymbol{F}_{0} ; u_{1}, u_{2} ; u_{3}, u_{4}\right)$ is called a $k$-fence if the following conditions (F14) are true:

(F1) For $1 \leq i, j \leq k$ and $|i-j| \geq 2$, the cycles $C_{i}, C_{j}$ are vertex-disjoint. Moreover, $u_{1}, u_{2} \notin V\left(C_{i}\right)$ for $i>1$, and $u_{3}, u_{4} \notin V\left(C_{i}\right)$ for $i<k$.

(F2) The graph $\boldsymbol{F}_{0}=C_{1} \cup \ldots \cup C_{k}$ is connected and planar.

Let $n=1,2$. We define a set $X_{n} \subset V\left(\boldsymbol{F}_{0}\right)$ recursively as follows: $u_{n} \in X_{n}$; and, for $i=1,2, \ldots, k-1$ and $j=i+1$, if $x \in X_{n} \cap V\left(C_{i}\right), x^{\prime} \in V\left(C_{i}\right) \cap V\left(C_{j}\right)$ are such that there is a path $P \subset C_{i}$ with ends $x, x^{\prime}$ internally disjoint from $C_{j}$, then we add $x^{\prime}$ into $X_{n}$. We define sets $X_{n}, n=3,4$ analogously for $i=k, k-1, \ldots, 2$ and $j=i-1$.

(F3) For $n=1,2$ (for $n=3,4)$ and $2 \leq i \leq k-1$ the next holds: if $P \subset C_{i}$ is a path with both ends in $X_{n} \cap V\left(C_{i}\right) \cap V\left(C_{i-1}\right)$ (in $X_{n} \cap V\left(C_{i}\right) \cap V\left(C_{i+1}\right)$ ), then $P$ intersects $V\left(C_{i+1}\right)$ ( $P$ intersects $V\left(C_{i-1}\right)$ ).

(F4) The sets $X_{1}, X_{2}, X_{3}, X_{4}$ are pairwise disjoint. For $1 \leq i \leq k$; if $v_{n} \in$ $X_{n} \cap V\left(C_{i}\right), n=1,2,3,4$, then the vertices $v_{1}, v_{3}, v_{2}, v_{4}$ lie in this cyclic order on the cycle $C_{i}$.

Moreover, a graph $\boldsymbol{F}$ is called a crossed $k$-fence if $\boldsymbol{F}=\boldsymbol{F}_{0} \cup Q_{1} \cup Q_{2} \cup Q$ and $u_{1}, u_{2}, u_{3}, u_{4}$ are such that the following is true:

(F5) $\boldsymbol{F}_{0}$ is a graph, $u_{1}, u_{2}, u_{3}, u_{4} \in V\left(\boldsymbol{F}_{0}\right)$, and $\left(\boldsymbol{F}_{0} ; u_{1}, u_{2} ; u_{3}, u_{4}\right)$ is a $k$-fence. 


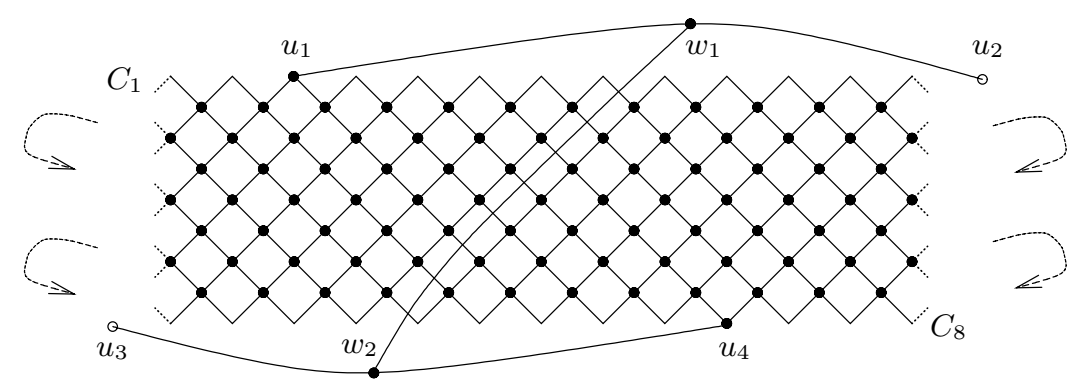

Fig. 3. A basic example of a crossed 8-fence. (The "fence" is winded on a cylinder.)

(F6) $Q_{1}$ is a path with ends $u_{1}, u_{2}$ internally disjoint from $\boldsymbol{F}_{0}$, and $Q_{2}$ is a path with ends $u_{3}, u_{4}$ internally disjoint from $\boldsymbol{F}_{0} \cup Q_{1}$. For some internal vertices $w_{1} \in V\left(Q_{1}\right), w_{2} \in V\left(Q_{2}\right)$ of the paths $Q_{1}, Q_{2}$, the path $Q$ connects $w_{1}, w_{2}$ and is internally disjoint from $\boldsymbol{F}_{0} \cup Q_{1} \cup Q_{2}$.

As an illustration to the above definition we present an example of a crossed 8-fence in Fig. 3. We also add some informal comments to the definition: By the definition, the graph $\boldsymbol{F}_{0}$ is planar. Moreover, it immediately follows from (F12) that we may draw $\boldsymbol{F}_{0}$ without crossings as a "bunch of concentric cycles", i.e. each cycle $C_{i}$ is a closed curve separating $C_{1} \cup \ldots \cup C_{i-1}$ from $C_{i+1} \cup \ldots \cup$ $C_{k}$. (See also Fig. 4) Notice that the definition of a fence $\left(\boldsymbol{F}_{0} ; u_{1}, u_{2} ; u_{3}, u_{4}\right)$ is symmetric with respect to any one of $u_{1}, u_{2}, u_{3}, u_{4}$ (possibly reversing the order of cycles in $\left.\boldsymbol{F}_{0}\right)$. Notice also that the sets $X_{n}, n=1,2,3,4$ intersect all cycles of $\boldsymbol{F}_{0}$. More properties of a fence are illustrated by two easy lemmas.

Lemma 4.1. Let $\left(\boldsymbol{G}_{0} ; u_{1}, u_{2} ; u_{3}, u_{4}\right)$ be a $k$-fence, $k \geq 2$, where $\boldsymbol{G}_{0}=C_{1} \cup C_{2} \cup$ $\ldots \cup C_{k}$. We denote by $\boldsymbol{G}_{0}^{\prime}=C_{2} \cup C_{3} \cup \ldots \cup C_{k}$, and by $u_{i}^{\prime}, i=1,2$ some vertex of $C_{1} \cap C_{2}$ such that there is a path $P_{i} \subset C_{1}$ with ends $u_{i}, u_{i}^{\prime}$ internally disjoint from $C_{2}$. Then $\left(\boldsymbol{G}_{0}^{\prime} ; u_{1}^{\prime}, u_{2}^{\prime} ; u_{3}, u_{4}\right)$ is a $(k-1)$-fence.

Proof. Let us look at the definition of a fence on page 108. The conditions (F12 ) from the definition are clearly satisfied for $\boldsymbol{G}_{0}^{\prime}$. In particular, $u_{1}^{\prime}, u_{2}^{\prime} \notin V\left(C_{i}\right)$ for $i>2$ since $u_{1}^{\prime}, u_{2}^{\prime} \in V\left(C_{1}\right)$. We denote by $X_{n}^{\prime}, n=1,2,3,4$ the sets defined analogously to $X_{n}$ for $G_{0}^{\prime}$. Then $X_{n}^{\prime}=X_{n} \backslash V\left(C_{1}\right)$ for $n=3,4$, and $X_{n}^{\prime} \subset X_{n}$ for $n=1,2$ since $u_{n}^{\prime} \in X_{n}$ by the definition. So validity of the conditions (F3-4) for $\boldsymbol{G}_{0}^{\prime}$ follows easily, and $\boldsymbol{G}_{0}^{\prime}$ forms a $(k-1)$-fence.

Lemma 4.2. Let $\boldsymbol{G}$ be a crossed $k$-fence, $k \geq 1$. Then $\operatorname{cr}(\boldsymbol{G}) \leq k$, and $\operatorname{cr}(\boldsymbol{G}-e) \leq k-1$ for all edges $e \in E(\boldsymbol{G})$.

Proof. This is an easy proof again, so we only sketch it. (See the scheme in Fig. 4) We use the notation $\boldsymbol{G}=\boldsymbol{G}_{0} \cup Q_{1} \cup Q_{2} \cup Q$ and $\boldsymbol{G}_{0}=C_{1} \cup \ldots \cup C_{k}$ analogously to the definition of a crossed fence. As noted above, $\boldsymbol{G}_{0}$ can be 


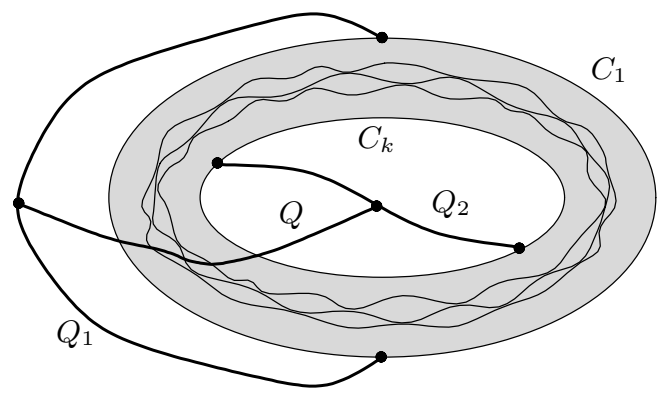

Fig. 4. A generic plane drawing of a crossed $k$-fence.

drawn without crossings as a "bunch" of concentric cycles. We may add the paths $Q_{1}, Q_{2}$ "outside" and "inside" to $\boldsymbol{G}_{0}$ again without crossings. Finally, we draw the path $Q$ connecting a vertex of $Q_{1}$ to a vertex of $Q_{2}$ so that it crosses each of the $k$ cycles $C_{i}$ of $\boldsymbol{G}_{0}$ exactly once.

Next, we show how to modify the previous drawing of $\boldsymbol{G}$ to get a drawing of $\boldsymbol{G}-e$ with less than $k$ crossings: If $e \in E\left(\boldsymbol{G}_{0}\right)$, then we may avoid the crossing of $Q$ with the cycle $C_{j}, e \in E\left(C_{j}\right)$. If $e \in E(Q)$, then $\boldsymbol{G}-e$ is planar. Lastly, if $e \in E\left(Q_{1}\right)$ (which is symmetric to $e \in E\left(Q_{2}\right)$ ), then we may redraw $\left(Q \cup Q_{1}\right)-e$ so that it does not cross $C_{1}$.

Lemma 4.3. Let $\boldsymbol{G}$ be a crossed $k$-fence, $k=1$ or $k \geq 3$. Then $\operatorname{cr}(\boldsymbol{G}) \geq k$.

Proof. We use induction on $k$. In the base case $k=1, \boldsymbol{G}$ is a subdivision of the nonplanar graph $K_{3,3}$, and so $\operatorname{cr}(\boldsymbol{G})=1$. Unfortunately, our statement is false for $k=2$; a crossed 2-fence may have crossing number 1 . Thus we must avoid referring to that case in the induction. We first present a general inductive step, and then we show how to overcome the exceptional value of 2 .

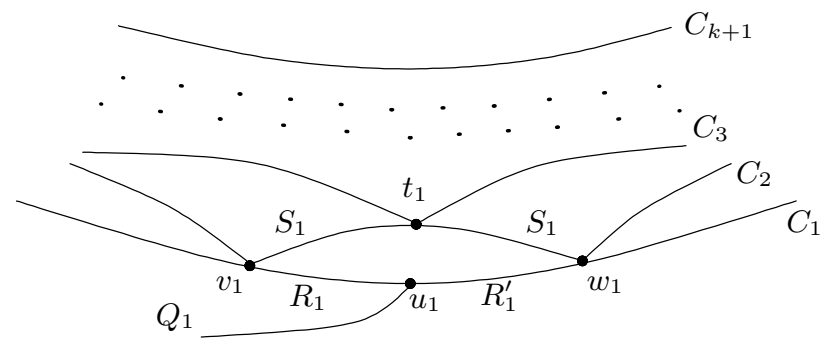

Fig. 5. An illustration to the proof.

Let us have an optimal drawing $\boldsymbol{H}$ of a crossed $(\mathrm{k}+1)$-fence, where $\boldsymbol{H}=$ $\boldsymbol{H}_{0} \cup Q_{1} \cup Q_{2} \cup Q$ and $\boldsymbol{H}_{0}=C_{1} \cup \ldots \cup C_{k+1}, u_{1}, u_{2} \in V\left(C_{1}\right), u_{3}, u_{4} \in V\left(C_{k+1}\right)$, 
as in the definition of a crossed fence. By connectivity of $\boldsymbol{H}_{0}$, there are two (possibly equal for now) vertices $v_{1}, w_{1} \in V\left(C_{1}\right) \cap V\left(C_{2}\right)$ such that the edgedisjoint paths $R_{1}, R_{1}^{\prime} \subset C_{1}$ connecting $u_{1}$ to $v_{1}$ and $u_{1}$ to $w_{1}$, resp., are internally disjoint from $C_{2}$. (See Fig. 5) We define vertices $v_{2}, w_{2}$ and paths $R_{2}, R_{2}^{\prime} \subset C_{1}$ analogously for $u_{2}$. Then $v_{1}, w_{1} \in X_{1}$ and $v_{2}, w_{2} \in X_{2}$ by the definition. It follows from (F4) that $v_{1}, w_{1}, v_{2}, w_{2}$ are pairwise distinct.

We first assume that some edge $e \in E\left(C_{1}\right)$ is crossed in $\boldsymbol{H}$. Up to symmetry, we may assume that $e \notin E\left(R_{1}\right)$ and $e \notin E\left(R_{2}\right)$. We set $\boldsymbol{H}_{0}^{\prime}=C_{2} \cup C_{3} \cup \ldots \cup C_{k+1}$, $u_{1}^{\prime}=v_{1}, u_{2}^{\prime}=v_{2}$. By Lemma 4.1 $\left(\boldsymbol{H}_{0}^{\prime} ; u_{1}^{\prime}, u_{2}^{\prime} ; u_{3}, u_{4}\right)$ is a $k$-fence; and hence $\boldsymbol{H}^{\prime}=\boldsymbol{H}_{0}^{\prime} \cup\left(Q_{1} \cup R_{1}\right) \cup\left(Q_{2} \cup R_{2}\right) \cup Q$ is a crossed $k$-fence. However, the drawing $\boldsymbol{H}^{\prime}$ has at least one crossing less than $\boldsymbol{H}$ since $e \notin E\left(\boldsymbol{H}^{\prime}\right)$. Therefore, $\operatorname{cr}(\boldsymbol{H}) \geq$ $\operatorname{cr}\left(\boldsymbol{H}^{\prime}\right)+1 \geq k+1$ if $k \neq 2$.

Second, we assume that no edge of $C_{1}$ is crossed in $\boldsymbol{H}$. We denote by $S_{1} \subset C_{2}$ the path with ends $v_{1}, w_{1}$ and disjoint from $v_{2}, w_{2}$; and $S_{2} \subset C_{2}$ with ends $v_{2}, w_{2}$ analogously. By (F3), both paths $S_{1}, S_{2}$ intersect the cycle $C_{3}$. Moreover, since $C_{3} \cup \ldots \cup C_{k+1} \cup Q_{1} \cup Q_{2} \cup Q$ is a connected graph, all three paths $S_{1}, S_{2}, Q_{1}$ are drawn in the same region of $C_{1}$ by the Jordan Curve Theorem. (Recall that $C_{1}$ is drawn as an uncrossed closed curve.) It follows from the order of the path ends on $C_{1}$ that the path $Q_{1}$ must cross both paths $S_{1}, S_{2}$, say in edges $e_{1} \in E\left(S_{1}\right)$, $e_{2} \in E\left(S_{2}\right)$. We denote by $t_{n} \in V\left(S_{n}\right) \cap V\left(C_{3}\right), n=1,2$ vertices such that (F3) there are subpaths $S_{n}^{\prime} \subset S_{n}-e_{n}$ connecting $v_{n}$ (or $w_{n}$, up to symmetry) to $t_{n}$ and internally disjoint from $C_{3}$. We set $\boldsymbol{H}_{0}^{\prime \prime}=C_{3} \cup \ldots \cup C_{k+1}, u_{1}^{\prime \prime}=t_{1}, u_{2}^{\prime \prime}=t_{2}$. Then $\left(\boldsymbol{H}_{0}^{\prime \prime} ; u_{1}^{\prime \prime}, u_{2}^{\prime \prime} ; u_{3}, u_{4}\right)$ is a $(k-1)$-fence by double application of Lemma 4.1 and so $\boldsymbol{H}^{\prime \prime}=\boldsymbol{H}_{0}^{\prime \prime} \cup\left(Q_{1} \cup R_{1} \cup S_{1}^{\prime}\right) \cup\left(Q_{2} \cup R_{2} \cup S_{2}^{\prime}\right) \cup Q$ is a crossed $(k-1)$-fence. Therefore, $\operatorname{cr}(\boldsymbol{H}) \geq \operatorname{cr}\left(\boldsymbol{H}^{\prime \prime}\right)+2 \geq k+1$ if $k-1 \neq 2$.

Finally, we resolve the exceptions left above. Suppose that $k=2$ in the first case, and that the second case cannot be symmetrically applied (i.e. $C_{3}$ is crossed as well). Then we may actually repeat this step twice (for $C_{1}$ and $C_{3}$ in $\boldsymbol{H}$ ), and refer to the inductive assumption for $k-1=1$. Suppose that $k=3$ in the second case, and that the first case cannot be symmetrically applied (i.e. neither $C_{4}$ is crossed). Then again, we argue twice in the same way, showing that both cycles $C_{2}, C_{3}$ of $\boldsymbol{H}$ are crossed at least twice each by the paths $Q_{1}, Q_{2}$, resp. Hence $\operatorname{cr}(\boldsymbol{H}) \geq 2+2=4$ in this case, as desired.

The previous Lemmas 4.24 .3 immediately imply:

Theorem 4.4. Let $\boldsymbol{G}$ be a crossed $k$-fence, $k=1$ or $k \geq 3$. Then $\boldsymbol{G}$ is a $k$ crossing-critical graph.

\section{Lower Bounds}

In this section we are going to prove Theorem 2.5 by exhibiting crossed fences that contain large binary trees. (The example of a crossed $k$-fence from Fig. 3 contains a subdivision of a binary tree of height about $\frac{k}{2}$, however, we provide even better constructions now.) 
Lemma 5.1. There exists a graph $\boldsymbol{H}^{k}, k \geq 1$ such that $\boldsymbol{H}^{k}$ is a crossed $k$-fence, and that $\boldsymbol{H}^{k}$ contains a binary tree of height $k+2$.

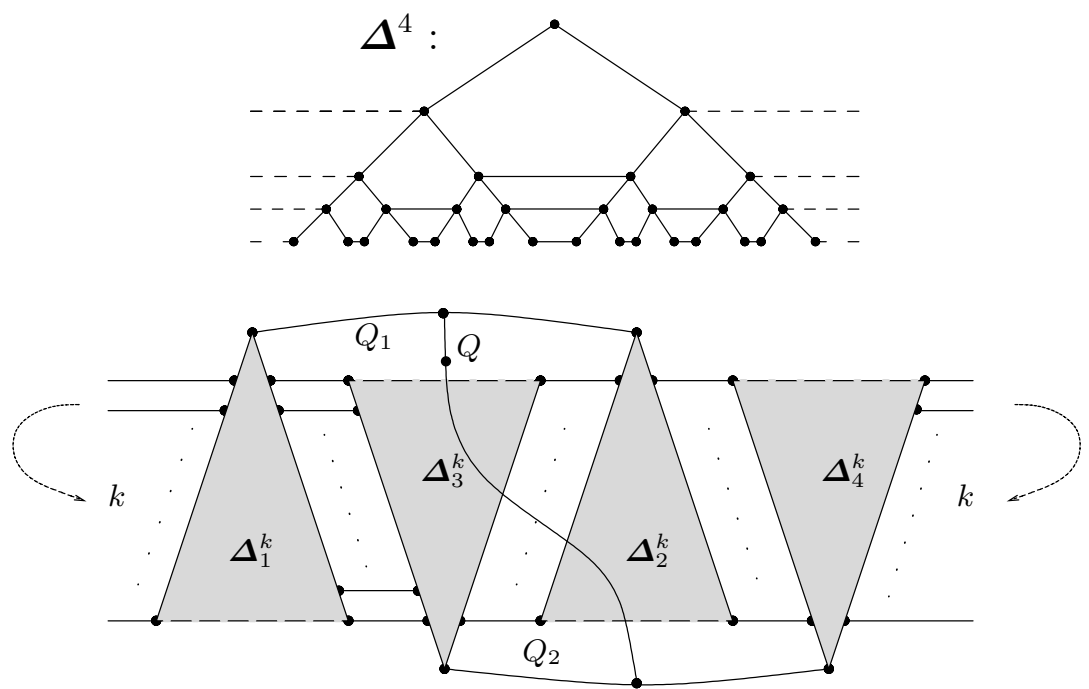

Fig. 6. A scheme of the construction of graph $\boldsymbol{H}^{k}$ (cf. Lemma 5.1).

Proof. We denote by $\boldsymbol{\Delta}^{k}$ a graph described as follows: The vertex set of $\boldsymbol{\Delta}^{k}$ consists of all words starting with a symbol $r$ and appending a sequence of at most $k$ symbols chosen from 0,1 . Each vertex (word) $\langle x\rangle$ of $\boldsymbol{\Delta}^{k}$ is adjacent to both $\langle x 0\rangle,\langle x 1\rangle$ (unless $x$ is longer than $k$ ). Moreover, each vertex of pattern $\left\langle y 01^{i}\right\rangle, i \geq 1$ in $\boldsymbol{\Delta}^{k}$ is adjacent to $\left\langle y 10^{i}\right\rangle$. (The exponent of a symbol counts repetition of this symbol in the word.) The construction is illustrated by an example of $\boldsymbol{\Delta}^{4}$ on the top of Fig. 6] Clearly, $\boldsymbol{\Delta}^{k}$ has a spanning binary tree with the root $\langle r\rangle$.

A graph $\boldsymbol{H}_{0}^{k}$ is a disjoint union of four copies $\boldsymbol{\Delta}_{1}^{k}, \boldsymbol{\Delta}_{2}^{k}, \boldsymbol{\Delta}_{3}^{k}, \boldsymbol{\Delta}_{4}^{k}$ of $\boldsymbol{\Delta}^{k}$ joined by edges in the following way: For $i=1, \ldots, k$, a vertex $\left\langle r 0^{i}\right\rangle$ of $\boldsymbol{\Delta}_{1}^{k}$ is adjacent to a vertex $\left\langle r 0^{k+1-i}\right\rangle$ of $\boldsymbol{\Delta}_{4}^{k}$; and a vertex $\left\langle r 1^{i}\right\rangle$ of $\boldsymbol{\Delta}_{1}^{k}$ is adjacent to a vertex $\left\langle r 1^{k+1-i}\right\rangle$ of $\boldsymbol{\Delta}_{3}^{k}$. Vertices of $\boldsymbol{\Delta}_{2}^{k}$ are analogously adjacent to $\boldsymbol{\Delta}_{3}^{k}$ and to $\boldsymbol{\Delta}_{4}^{k}$. Fig. 6 shows a scheme of the construction. We claim that $\boldsymbol{H}_{0}^{k}$ is a $k$-fence (see the definition on page 108): It is easy to see the cycles $C_{i}$ - the vertex set $V\left(C_{i}\right)$ is formed by all words in $\boldsymbol{\Delta}_{1}^{k}, \boldsymbol{\Delta}_{2}^{k}$ of length $i$ or $i+1$, and by all words in $\boldsymbol{\Delta}_{3}^{k}$, $\boldsymbol{\Delta}_{4}^{k}$ of length $k+2-i$ or $k+1-i$. The vertices $u_{1}, u_{2}, u_{3}, u_{4}$ from the definition are the respective roots, and the sets $X_{1}, X_{2}, X_{3}, X_{4}$ are the respective vertex sets, of $\boldsymbol{\Delta}_{1}^{k}, \boldsymbol{\Delta}_{2}^{k}, \boldsymbol{\Delta}_{3}^{k}, \boldsymbol{\Delta}_{4}^{k}$.

The graph $\boldsymbol{H}^{k}$ results from $\boldsymbol{H}_{0}^{k}$ by adding paths $Q_{1}, Q_{2}$ and $Q$, each of length two, as required by the definition of a crossed fence. Then $\boldsymbol{H}^{k}$ has a spanning binary tree of height $k+2$, the root of which is the middle vertex of path $Q$. 
Lemma 5.2. There exists a simple 3 -connected graph $\tilde{\boldsymbol{H}}^{k}, k \geq 1$ such that $\tilde{\boldsymbol{H}}^{k}$ is a crossed $k$-fence, and $\tilde{\boldsymbol{H}}^{k}$ contains a binary tree of height $k-1$.

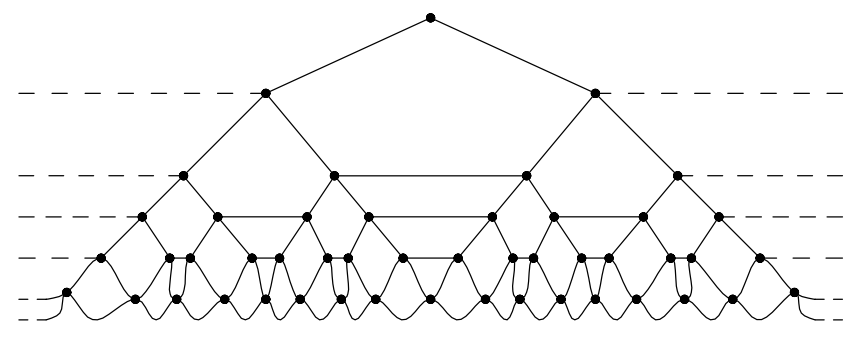

Fig. 7. A modified construction of a graph $\tilde{\Delta}^{6}$ (cf. Lemma 5.2).

Proof. The construction of $\tilde{\boldsymbol{H}}^{k}$ is almost the same as the previous construction of $\boldsymbol{H}^{k}$, but we use copies of a graph $\tilde{\boldsymbol{\Delta}}^{k}$ instead of $\boldsymbol{\Delta}^{k}$. Simply speaking, $\tilde{\boldsymbol{\Delta}}^{k}$ is obtained from $\boldsymbol{\Delta}^{k-2}$ by adding two paths that "form a lace on the bottom vertices", as shown in Fig. 7. Rest follows the scheme in Fig. 6. with a minor variation that the path $Q$ now consists of one edge.

We formally describe the construction as follows: The graph $\tilde{\boldsymbol{\Delta}}^{k}$ results from $\Delta^{k-1}$ (see in the proof of Lemma 5.1) by contracting all edges of the pattern $\left\{\left\langle y 01^{i}\right\rangle,\left\langle y 10^{i}\right\rangle\right\}$ where $y$ is a prefix of length $k-1-i$, and by adding all edges of the pattern $\{\langle z 0\rangle,\langle z 1\rangle\}$ where $z$ is a prefix of length $k-1$. Then $\tilde{\boldsymbol{H}}_{0}^{k}$ is constructed from four copies $\tilde{\boldsymbol{\Delta}}_{1}^{k}, \tilde{\boldsymbol{\Delta}}_{2}^{k}, \tilde{\boldsymbol{\Delta}}_{3}^{k}, \tilde{\boldsymbol{\Delta}}_{4}^{k}$ of $\tilde{\boldsymbol{\Delta}}^{k}$. A vertex $\left\langle r 0^{i}\right\rangle, i=2, \ldots, k-1$ of $\tilde{\boldsymbol{\Delta}}_{1}^{k}$ is adjacent to a vertex $\left\langle r 0^{k+1-i}\right\rangle$ of $\tilde{\boldsymbol{\Delta}}_{3}^{k}$, a vertex $\langle r 0\rangle$ of $\tilde{\boldsymbol{\Delta}}_{1}^{k}$ is adjacent to $\left\langle r 0^{k-1}\right\rangle$ of $\tilde{\boldsymbol{\Delta}}_{3}^{k}$, and $\left\langle r 0^{k-1}\right\rangle$ of $\tilde{\boldsymbol{\Delta}}_{1}^{k}$ is adjacent to $\langle r 0\rangle$ of $\tilde{\boldsymbol{\Delta}}_{3}^{k}$. Analogously, vertices of $\tilde{\boldsymbol{\Delta}}_{1}^{k}$ are adjacent to vertices of $\tilde{\boldsymbol{\Delta}}_{4}^{k}$, and vertices of $\tilde{\boldsymbol{\Delta}}_{2}^{k}$ are adjacent to vertices of $\tilde{\boldsymbol{\Delta}}_{3}^{k}$ and of $\tilde{\boldsymbol{\Delta}}_{4}^{k}$.

It is a routine work to verify that $\tilde{\boldsymbol{H}}^{k}$ is a simple 3 -connected graph and a crossed $k$-fence. The largest binary tree in $\tilde{\boldsymbol{H}}^{k}$ spans $\tilde{\boldsymbol{\Delta}}_{1}^{k} \cup \tilde{\boldsymbol{\Delta}}_{2}^{k} \cup Q_{1}$ and it has height $k-1$.

Using Theorem 4.4 the proof of Theorem 2.5 is now finished.

\section{Conclusions}

We have shown polynomial lower and upper bounds on the height $f(k)$ of a subdivision of a largest binary tree that may be contained in a $k$-crossing-critical graph. Unfortunately, these bounds are still far apart. We do not make any conjecture about the correct asymptotic for the function $f(k)$ from Theorem 2.3 but we think that it would be closer to the linear lower bound than to the cubic upper bound. 


\section{References}

1. M. Ajtai, V. Chvátal, M.M. Newborn, E. Szemerédi, Crossing-free subgraphs., Theory and practice of combinatorics, 9-12, North-Holland Math. Stud. 60, NorthHolland, Amsterdam-New York, 1982.

2. D. Bienstock, N. Robertson, P. Seymour, R. Thomas, Quickly excluding a forest, J. Combin. Theory Ser. B 52 (1991), 274-283.

3. S.N. Bhatt, F.T. Leighton, A frame for solving VLSI graph layout problems, J. of Computer and Systems Science 28 (1984), 300-343.

4. G. Di Battista, P. Eades, R. Tamassia, I.G. Tollis, Graph Drawing: Algorithms for the Visualization of Graphs, Prentice Hall 1999 (ISBN 0-13-301615-3).

5. G. Ding, B. Oporowski, R. Thomas, D. Vertigan, Large four-connected nonplanar graphs, in preparation.

6. M.R. Garey, D.S. Johnson, Crossing number is NP-complete, SIAM J. Algebraic Discrete Methods 4 (1983), 312-316.

7. J. Geelen, B. Richter, G. Salazar, Embedding grids on surfaces, manuscript.

8. L.Y. Glebsky, G. Salazar, The conjecture $\operatorname{cr}\left(C_{m} \times C_{n}\right)=(m-2) n$ is true for all but finitely $n$, for each $m$, submitted.

9. P. Hliněný, Crossing-number critical graphs have bounded path-width, submitted. http://www .mcs. vuw.ac.nz/ ^hlineny/doc/crpath2.ps.gz

10. M. Kochol, Construction of crossing-critical graphs, Discrete Math. 66 (1987), 311313.

11. F.T. Leighton, Complexity Issues in VLSI, M.I.T. Press, Cambridge, 1983.

12. P. Mutzel, T. Ziegler, The Constrained Crossing Minimization Problem, In: Proceedings Graph Drawing '99, Štiřín Castle, Czech Republic, September 1999 (J. Kratochvíl ed.), 175-185; Lecture Notes in Computer Science 1731, Springer Verlag, Berlin 2000 (ISBN 3-540-66904-3).

13. J. Pach, G. Tóth, Which crossing number is it, anyway?, Proc. 39th Foundations of Computer Science (1998), IEEE Press 1999, 617-626.

14. H. Purchase, Which Aesthetics has the Greates Effect on Human Understanding, In: Proceedings Graph Drawing '97, Rome, Italy, September 18-20 1997 (G. DiBattista ed.), 248-261; Lecture Notes in Computer Science 1353, Springer Verlag, Berlin 1998 (ISBN 3-540-63938-1).

15. R.B. Richter, C. Thomassen, Intersections of curve systems and the crossing number of $C_{5} \times C_{5}$, Discrete Comput. Geom. 13 (1995), 149-159.

16. R.B. Richter, G. Salazar, The crossing number of $C_{6} \times C_{n}$, Australas. J. Combin. 23 (2001), 135-143.

17. N. Robertson, P. Seymour, Graph minors I. Excluding a forest, J. Combin. Theory Ser. B 35 (1983), 39-61.

18. F. Shahrokhi, I. Vrt'o, On 3-Layer Crossings and Pseudo Arrangements, In: Proceedings Graph Drawing '99, Śtiřín Castle, Czech Republic, September 1999 (J. Kratochvíl ed.), 225-231; Lecture Notes in Computer Science 1731, Springer Verlag, Berlin 2000 (ISBN 3-540-66904-3).

19. W.T. Tutte, Toward a theory of crossing numbers, J. Combinatorial Theory 8 (1970), 45-53. 